\title{
Color Analysis for the Quantitative Aesthetics of Qiong Kiln Ceramics
}

\author{
Fei Wang ${ }^{1,3}$, Hang Chao ${ }^{2,3}$, Lu Leng ${ }^{1,3 *}$
}

\begin{abstract}
The subjective experience would degrade the current artificial artistic aesthetic analysis. Since Qiong kiln ceramics have a long history and occupy a very important position in ceramic arts, we employed computer-aided technologies to quickly automatically accurately and quantitatively process a large number of Qiong kiln ceramic images and generate the detailed statistical data. Because the color features are simple and significant visual characteristics, the color features of Qiong kiln ceramics are analyzed for the quantitative aesthetics. The Qiong kiln ceramic images are segmented with GrabCut algorithm. Three moments (1st-order, 2nd-order, and 3rd-order) are calculated in two typical color spaces, namely RGB and HSV. The discrimination powers of the color features are analyzed according to various dynasties (Tang Dynasty, Five Dynasties, Song Dynasty) and various utensils (Pot, kettle, bowl), which are helpful to the selection of the discriminant color features among various dynasties and utensils. This paper is helpful to promoting the quantitative aesthetic research of Qiong kiln ceramics and is also conducive to the research on the aesthetics of other ceramics.
\end{abstract}

Key Words: Qiong kiln ceramics, Quantitative aesthetics, Color analysis, Discrimination power analysis.

\section{INTRODUCTION}

Qiong kiln ceramics were produced in many places, such as Sichuan and Southwest in China. They sprang up in the Five Dynasties, but their manufacturing sizes became smaller in the Song Dynasty. They were sold well in Chengdu (the capital of Sichuan province) and its surrounding areas. Figure 1 shows the Qiong kiln ceramics samples of various dynasties, namely Tang Dynasty (AD 681-AD 907), Five Dynasties (AD 907-AD 960), Song Dynasty (AD 960-AD 1279), and various utensils, namely pot, kettle, bowl.

In recent years, digital image processing and computer vision technologies have been applied to analyze the features [1], such as texture, color, and shape, which can also be used for the quantitative aesthetics of archeological ceramics. Compared with manual processing, computeraided technologies can quickly automatically accurately and quantitatively process a large number of digital ceramic images and generate detailed statistical data, which avoids the disturbance of subjective experience [2].

Kampel and Sablatnig applied digital image processing to the automatic classification of archeological fragments [3]. Based on the correlation of the same ceramic sample in shape, material and color, they used the color characteristics of ceramic fragments to restore the fragments into a complete sample. Fusion can improve the performance in many aspects [4-6]; therefore, Smith et al. classified the thin-shell ceramics based on color and texture descriptors and reconstructed the complete ceramic

\footnotetext{
Manuscript received: March 23, 2020; Revised: April 3, 2020; Accepted: April 5, 2020. (ID No. JMIS-20M-03-012)

Corresponding Author (*): Lu Leng, Nanchang Hangkong University, 696 Fenghe South Avenue, Nanchang, 330063, P.

R. China, +86-791-86453251, leng@nchu.edu.cn

${ }^{1}$ Key Laboratory of Jiangxi Province for Image Processing and Pattern Recognition, Nanchang Hangkong University, Nanchang, 330063, P. R. China, \{1009685258, 2267690972\}@qq.com, leng@nchu.edu.cn

${ }^{2}$ Key laboratory of Network and Data Security Lab, University of Electronic Science and Technology of China, Sichuan, 610054, P. R. China

${ }^{3}$ School of Software, Nanchang Hangkong University, Nanchang 330063, P. R. China
} 
samples [7]. Kamiri et al. and Shire et al. used digital image processing to detect the defects on the ceramic surface [8, 9]. Their methods have low computation complexity and are effective. Penaranda et al. controlled the tile production by estimating the color and the proportion of each particle, accordingly the appearance of the produced tiles is visual uniformity [10]. Sun detected the defects of the ceramic of water taps and designed a real-time computer digital image dynamic detection system using charge-coupled device as the image acquisition sensor [11]. Elbehiery et al. utilized digital image processing to detect the colors and defects of the tile surface [12]. This method is helpful to the tile production and improves the homogeneity of various batches. Ebrahimzadeh and Hossienzadeh developed a system to classify the ceramics based on some discriminative features, including contrast and entropy [13]. The classifier was a trained multi-layer perceptron neural network. They changed the number of layers and debugged the parameters of the network to improve the network performance.

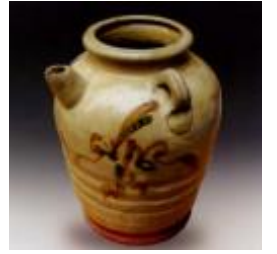

(a) Tang Dynasty.

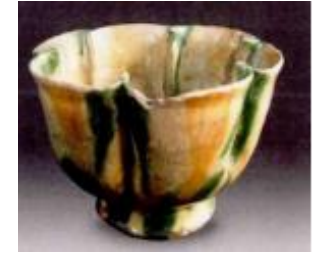

(b) Five Dynasties.

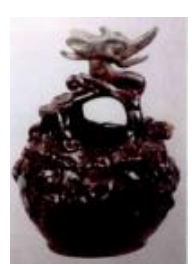

(c) Song Dynasty.

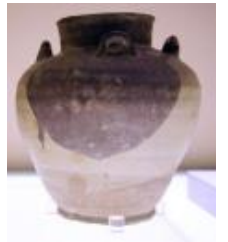

(d) Pot.

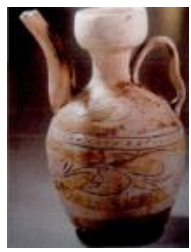

(e) Kettle.

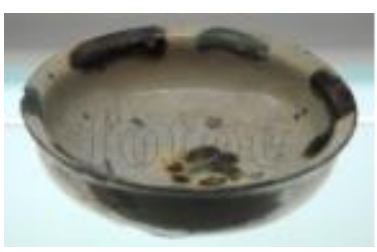

(f) Bowl.
Fig. 1. Qiong kiln ceramics samples.

The main contributions of this paper are as follows.

(1) Digital image processing and computer vision technologies are employed for the quantitative aesthetics of Qiong kiln ceramics. Since the color features are simple and significant visual characteristics, the color features of Qiong kiln ceramics are analyzed.

(2) Image segmentation is conducted to segment the foreground object from the background. Three moments (1st-order, 2nd-order, and 3rd-order) are calculated in two typical color spaces, namely RGB and HSV. The eighteen color features are analyzed according to various dynasties (Tang Dynasty, Five Dynasties, Song Dynasty) and various utensils (pot, kettle, bowl).

(3) The discrimination power (DP) of each feature is measured with discrimination power analysis (DPA) [14,
15], which are helpful to the selection of the discriminant color features among various dynasties and various utensils. The components with large DPs are more discriminative, which can improve classification accuracy.

The paper can help promote the quantitative aesthetic research of Qiong kiln ceramics and is conducive to the research on the aesthetics of other ceramics.

\section{METHODOLOGY}

\subsection{Image Segmentation}

Sine the color feature should be extracted and calculated only in the foreground region, i.e., the ceramic object region, rather than the background, object detection [16] and image segmentation [17], [21] are commonly used to separate the foreground object from the background. It is challenging for the segmentation of Qiong kiln ceramics since sometimes they have mixed and complex colors.

GrabCut algorithm is a famous image segmentation method based on graph cuts [18], which is suitable for Qiong kiln ceramic image. First a user specifies a bounding box around the ceramic object that needs to be segmented. The Gaussian mixture model is used for the estimation of the color distributions of the target object and background. A Markov random field is constructed over the pixel labels. The energy function prefers the connected regions that have the same label, and the optimization based on a graph cut is conducted to infer their values. Since it is likely that the estimation taken from the bounding box becomes more and more accurate, this procedure is repeated until convergence. If a user points out the misclassified regions and reruns the optimization, the estimation can be further corrected. In addition, GrabCut corrects the results to preserve edges.

In our dataset, each image contains only one ceramic object. Figure 2, the segmentation results based on GrabCut, demonstrates that GrabCut can accurately separate the ceramic object from the background.

\subsection{Color Space}

The color characteristics of Qiong kiln ceramics are analyzed in two color spaces, namely RGB (Red, Green, Blue) and HSV (Hue, Saturation, Value).

Let the three variables in $(r, g, b)$ denote the red, green, and blue components, respectively. Similarly, let the three variables in $(h, s, v)$ denote the hue, saturation, value components, respectively. max and min denote the maximum and minimum values of $r, g$ and $b$, respectively. Eqs. (1-3) realize the transformation from RGB space to 
HSV space; while Eqs. (4)-(9) realize the transformation from HSV space to RGB space.

$$
h= \begin{cases}0^{0} & \text { if } \max =\min \\ 60^{\circ} \times \frac{g-b}{\max -\min }+0^{\circ}, & \text { if } \max =r \text { and } g \geq b \\ 60^{\circ} \times \frac{g-b}{\max -\min }+360^{\circ}, \text { if } \max =r \text { and } g<b \\ 60^{\circ} \times \frac{g-b}{\max -\min }+120^{\circ}, \text { if } \max =g \\ 60^{\circ} \times \frac{g-b}{\max -\min }+240^{\circ}, \text { if } \max =b\end{cases}
$$$$
s= \begin{cases}0, & \text { if } \max =0 \\ \frac{\max -\min }{\max }=1-\frac{\min }{\max }, & \text { otherwise }\end{cases}
$$

$$
v=\max
$$

$$
h_{i}=\left\lfloor\frac{h}{60}\right\rfloor \quad(\bmod 6)
$$

$$
f=\frac{h}{60}-h_{i}
$$

$$
p=v \times(1-s)
$$

$$
q=v \times(1-f \times s)
$$

$$
t=v \times(1-(1-f) \times s)
$$

$$
(r, g, b)= \begin{cases}(v, t, p), & \text { if } h_{i}=0 \\ (q, v, p), & \text { if } h_{i}=1 \\ (p, v, t), & \text { if } h_{i}=2 \\ (p, q, v), & \text { if } h_{i}=3 \\ (t, p, v), & \text { if } h_{i}=4 \\ (v, p, q), & \text { if } h_{i}=5\end{cases}
$$

Figures 3 and 4 show the color component characteristics of a Qiong kiln ceramic image in HSV and RGB spaces, respectively. Figure 5 shows the histograms of the six color components. To facilitate the analysis and comparison, the ranges of all color components are normalized to [0,255].

\subsection{Moments}

Color moments are simple and effective color features. Since the color distribution characteristics mainly focus on the low-order moments, only the 1st-order, 2nd-order and 3rd-order moments are used as the color features.

1st-order color moment is the average value of the color component. The 1st-order color moment of the $i$-th component is:

$$
M_{i}=\frac{1}{N} \sum_{j=1}^{N} p_{i j},
$$

where $N$ is the number of pixels in the image, $p_{i j}$ is the $i$-th color component value of the $\mathrm{j}$-th pixel.
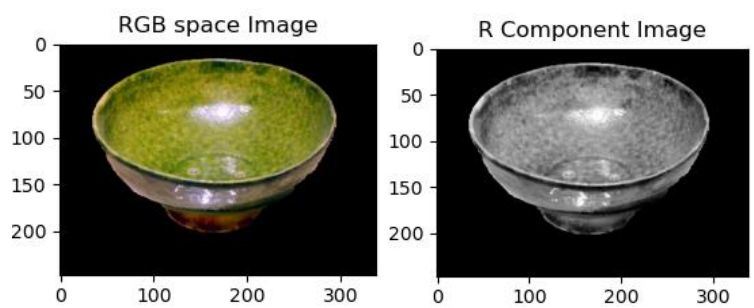

(a) RGB image

(b) R component image
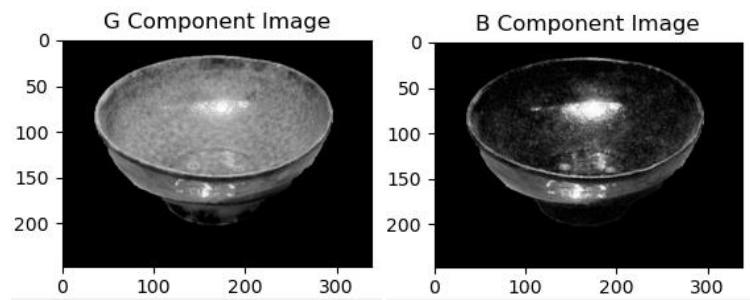

(c) $G$ component image

(d) B component image
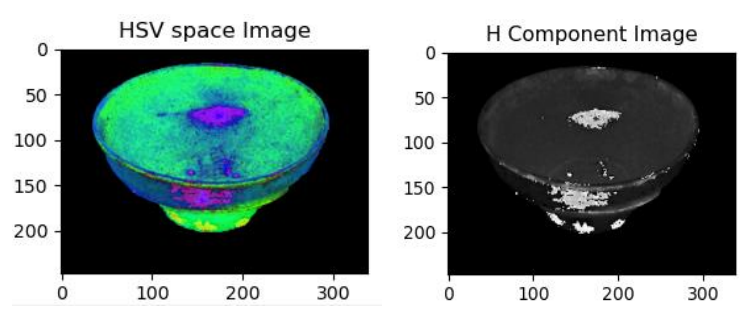

(a) HSV image

(b) H component image

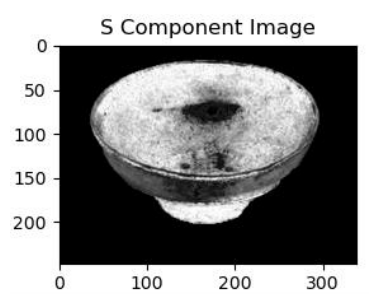

(c) S component image

(d) V component image

Fig. 4. HSV components. 


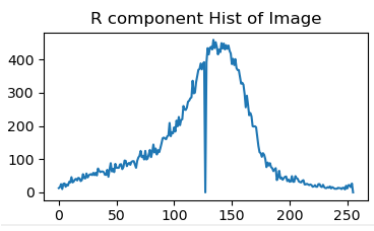

(a) $\mathrm{R}$ component

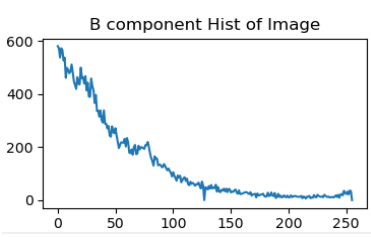

(c) B component

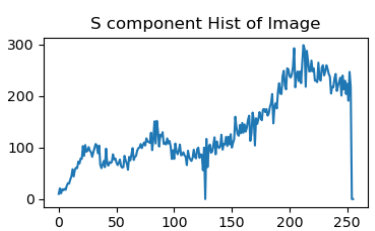

(e) S component

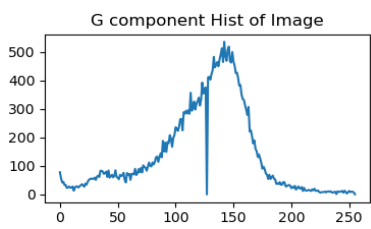

(b) G component

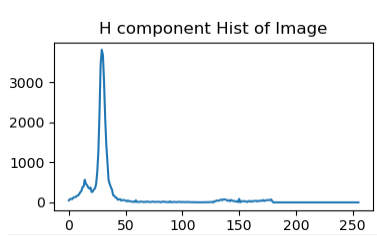

(d) H component

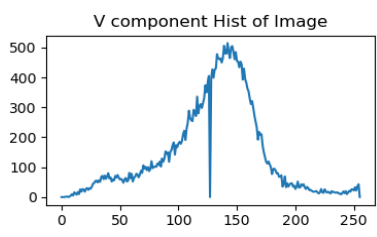

(f) $\mathrm{V}$ component
Fig. 5. Histograms of the color components.

\subsection{Moments}

Color moments are simple and effective color features. Since the color distribution characteristics mainly focus on the low-order moments, only the 1st-order, 2nd-order and 3rd-order moments are used as the color features.

1st-order color moment is the average value of the color component. The 1st-order color moment of the $i$-th component is:

$$
M_{i}=\frac{1}{N} \sum_{j=1}^{N} p_{i j}
$$

where $N$ is the number of pixels in the image, $p_{i j}$ is the $i$-th color component value of the $\mathrm{j}$-th pixel.

The 2nd-order color moment is the square root of the variance of the color distribution, i.e., standard deviation (STD), which measures how far the color component values are spread out from their average value. The 2nd-order color moment of the $i$-th component is:

$$
V_{i}=\sqrt{\left(\frac{1}{N} \sum_{j=1}^{N}\left(p_{i j}-M_{i}\right)^{2}\right)} .
$$

The 3rd-order color moment measures the lopsidedness, such as symmetry, of the color distribution. The 3rd-order color moment of the $i$-th component is:

$$
L_{i}=\sqrt[3]{\left(\frac{1}{N} \sum_{j=1}^{N}\left(p_{i j}-M_{i}\right)^{3}\right)} .
$$

Color Analysis for the Quantitative Aesthetics of Qiong Kiln Ceramics

Table 1 shows the three moments of the six color moments of the image in Figures 3 and 4.

Table 1. Color moments of one image sample.

\begin{tabular}{llll}
\hline & 1st-order & 2nd-order & 3rd-order \\
\hline R & 125.99 & 43.17 & -31.52 \\
G & 123.87 & 41.10 & -35.49 \\
B & 52.10 & 50.72 & 60.22 \\
H & 52.40 & 45.10 & 65.94 \\
S & 163.53 & 69.65 & -58.50 \\
V & 130.62 & 40.51 & -25.57 \\
\hline
\end{tabular}

\subsection{Discrimination Power Analysis}

DPA can measure the discrimination power (DP) of each feature. The larger the DP value is, the more discriminant the feature is. Assume that the dataset contains $C$ classes, and the number of the samples of the $i$-th class is $S_{i}$. DP of each color feature can be calculated as follows.

(1) $x_{i j}$ denotes the feature of the $j$-th sample of the $i$-th class.

(2) Calculate the average value of each class:

$$
m_{i}=\frac{1}{S_{j}} \sum_{j=1}^{S_{j}} x_{i j}
$$

(3) Calculate variance of each class:

$$
v_{i}=\frac{1}{S_{j}} \sum_{j=1}^{S_{j}}\left(x_{i j}-m_{i}\right)^{2} .
$$

(4) Calculate the average variance of the classes:

$$
v^{C}=\frac{1}{C} \sum_{i=1}^{C} v_{i} .
$$

(5) Calculate the average of all training samples:

$$
m^{A}=\frac{1}{\sum_{i=1}^{C} S_{i}} \sum_{i=1}^{C} \sum_{j=1}^{S_{i}} x_{i j}
$$

(6) Calculate the variance of all training samples: 


$$
v^{A}=\frac{1}{\sum_{i=1}^{C} S_{i}} \sum_{i=1}^{C} \sum_{j=1}^{S_{i}}\left(x_{i j}-m^{A}\right)
$$

(7) Calculate the DP of this feature:

$$
d p=\frac{v^{A}}{v^{C}}
$$

All the DPs of the eighteen color features can be measured with DPA to reveal their discriminant ability.

\section{EXPERIMENTAL RESULTS AND DISCUSSIONS}

The color features are labeled as one letter and one number, which denote the color component and moment order, respectively. Take an example, "R1" denotes the 1storder moment of red component. The color features of various dynasties and utensils are labeled as two letters and one number, which denote the dynasty/utensil, color component and moment order, respectively. Take an example, "TR1" denotes the 1st-order moment of red component of Tang dynasty.

Figures 6 and 7 show the distributions of the eighteen color features of the samples in the dataset. Tables 2 and 3 show the average color components of the samples in various dynasties and of various utensils, respectively.

In RGB space, R1>G1>B1 for all dynasties and utensils, which demonstrates the proportions of the three components. In contrast, R2, G2 and B2 are approximate for each dynasty or utensil. TG3, PG3, KG3 and BG3 are much smaller than the other 3rd-order moments, which reveal their high symmetry characteristics.

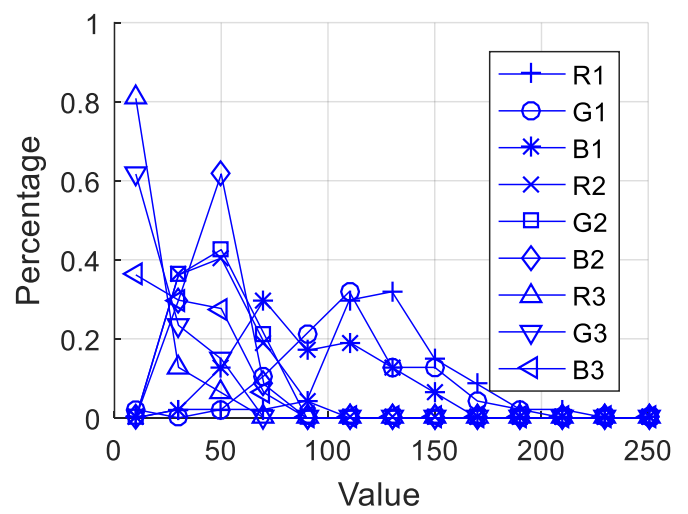

Fig. 6. Distributions of the color features in RGB color space.

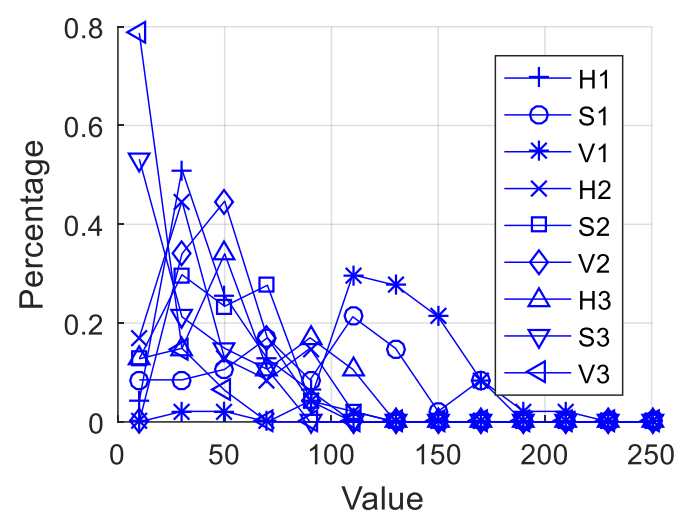

Fig. 7. Distributions of the color features in HSV color space.

(1) In RGB space, Five Dynasties has larger 2nd-order moments than Tang Dynasty; while Tang Dynasty has larger 2nd-order moments than Song Dynasty. Pot and Kettle have the similar 2nd-order moments that are larger than those of Bowl.

(2) In HSV space, according to $\mathrm{H} 1$ and $\mathrm{H} 2$, the colors are close to orange, yellow and green. According to S1 and $\mathrm{V} 1$, various dynasties and utensils can be compared in terms of saturation and brightness. TS3 is the smallest absolute 3rd-order moments for all dynasties. $|\mathrm{S} 3|<|\mathrm{V} 3|<|\mathrm{H} 3|$ for all utensils.

(3) Tables 4 and 5 show the STDs of the color components of the samples in various dynasties and of various utensils, respectively.

[1] In RGB space, generally, the 1st-order moments of Five Dynasties are larger than those of Song Dynasty. Bowl has larger 1st-order moments than Pot; while Pot has larger 1st-order moments than Kettle. Generally, $\mathrm{R} 2>\mathrm{G} 2>\mathrm{B} 2$ for all dynasties and utensils. Five Dynasties has larger 1st-order moments than the other two dynasties.

[2] In HSV, generally $\mathrm{S} 1>\mathrm{V} 1>\mathrm{H} 1$ for all dynasties. There is no remarkable regular pattern for 2nd-order moments. Generally, S3>V3>H3 for all dynasties and utensils.

Table 2. Means of color components in various dynasties.

\begin{tabular}{llll}
\hline & Tang & Five & Song \\
\hline R1 & 127.22 & 143.36 & 107.32 \\
G1 & 110.91 & 124.61 & 73.22 \\
B1 & 92.48 & 85.15 & 54.37 \\
R2 & 46.53 & 56.95 & 39.56 \\
G2 & 47.08 & 55.07 & 41.31 \\
B2 & 44.69 & 55.16 & 44.67 \\
\hline
\end{tabular}


Color Analysis for the Quantitative Aesthetics of Qiong Kiln Ceramics

\begin{tabular}{llll}
\hline R3 & -18.64 & -43.19 & 14.56 \\
G3 & -2.28 & -24.30 & 38.09 \\
B3 & 21.00 & 39.32 & 47.58 \\
H1 & 42.90 & 37.61 & 49.39 \\
S1 & 82.37 & 116.94 & 126.44 \\
V1 & 128.75 & 144.65 & 107.34 \\
H2 & 39.95 & 42.71 & 66.84 \\
S2 & 43.14 & 58.23 & 71.88 \\
V2 & 46.12 & 56.22 & 39.56 \\
H3 & 51.96 & 64.47 & 74.71 \\
S3 & 9.18 & -27.18 & 22.94 \\
V3 & -18.50 & -41.97 & 14.59 \\
\hline
\end{tabular}

Table 3. Means of color components of various utensils.

\begin{tabular}{llll}
\hline & Pot & Kettle & Bowl \\
\hline R1 & 128.91 & 130.41 & 116.33 \\
G1 & 105.15 & 113.98 & 102.55 \\
B1 & 88.16 & 94.01 & 74.20 \\
R2 & 48.76 & 48.31 & 41.14 \\
G2 & 50.02 & 48.71 & 40.19 \\
B2 & 45.78 & 47.33 & 40.50 \\
R3 & -27.13 & -17.68 & -13.26 \\
G3 & 1.43 & -2.37 & -5.69 \\
B3 & 21.54 & 24.68 & 28.78 \\
H1 & 45.29 & 41.76 & 42.70 \\
S1 & 101.39 & 80.98 & 99.20 \\
V1 & 130.24 & 131.37 & 119.45 \\
H2 & 59.05 & 39.49 & 27.11 \\
S2 & 55.74 & 44.02 & 42.95 \\
V2 & 47.70 & 48.30 & 40.16 \\
H3 & 75.22 & 51.43 & 38.20 \\
S3 & 7.76 & 4.39 & 10.33 \\
V3 & -26.86 & -17.65 & -12.24 \\
\hline
\end{tabular}

Tables 6 and 7 show the DPs of color components according to various dynasties and of various utensils, respectively. The larger the DP value is, the more discriminant the feature is.

(1) For dynasty discrimination, B2 is the most discriminant feature; while R2, H1, H3 are the lease discriminant features.

(2) For utensil discrimination, R3 and H1 are the most and least discriminant features, respectively.

(3) The DPs of the color features differ for various dynasties and for various utensils. All the DPs in Table 7 are close to 1; while the DPs in Table 8 fluctuate more dramatically.
Table 4. STDs of color components in various dynasties.

\begin{tabular}{llll}
\hline & Tang & Five & Song \\
\hline R1 & 22.96 & 36.14 & 25.97 \\
G1 & 30.33 & 33.83 & 13.78 \\
B1 & 28.38 & 34.31 & 15.46 \\
R2 & 11.90 & 16.42 & 17.73 \\
G2 & 9.93 & 14.79 & 15.57 \\
B2 & 9.57 & 13.21 & 15.08 \\
R3 & 27.91 & 37.09 & 30.43 \\
G3 & 38.95 & 36.68 & 30.04 \\
B3 & 30.36 & 30.90 & 27.47 \\
H1 & 21.49 & 20.12 & 23.61 \\
S1 & 38.83 & 44.97 & 51.45 \\
V1 & 22.08 & 35.91 & 22.65 \\
H2 & 28.86 & 26.53 & 17.24 \\
S2 & 21.75 & 25.37 & 21.06 \\
V2 & 10.54 & 16.53 & 17.76 \\
H3 & 28.36 & 30.92 & 24.58 \\
S3 & 42.17 & 43.47 & 42.46 \\
V3 & 28.24 & 37.43 & 29.44 \\
\hline
\end{tabular}

Table 5. STDs of color components of various utensils.

\begin{tabular}{llll}
\hline & Pot & Kettle & Bowl \\
\hline R1 & 29.85 & 15.57 & 67.78 \\
G1 & 29.02 & 15.33 & 47.30 \\
B1 & 30.17 & 28.48 & 31.65 \\
R2 & 15.54 & 17.15 & 10.94 \\
G2 & 14.27 & 13.31 & 11.54 \\
B2 & 13.11 & 8.30 & 10.38 \\
R3 & 33.28 & 15.03 & 39.68 \\
G3 & 35.29 & 28.39 & 9.68 \\
B3 & 29.62 & 31.43 & 5.34 \\
H1 & 19.96 & 10.68 & 42.91 \\
S1 & 44.55 & 36.50 & 31.38 \\
V1 & 28.81 & 14.27 & 67.75 \\
H2 & 26.79 & 11.33 & 48.80 \\
S2 & 23.64 & 13.43 & 26.40 \\
V2 & 15.43 & 17.38 & 10.94 \\
H3 & 32.89 & 13.00 & 26.81 \\
S3 & 40.50 & 33.53 & 63.64 \\
V3 & 33.53 & 16.30 & 39.63 \\
\hline
\end{tabular}

The DPs help to select the discriminant color features; however, DPA is greedy algorithm. The classification 
accuracy depends does not only on the DPs of the components, but also on their combination effects for fusion and dimensionality reduction [19-21]. We will study the fusion and dimensionality reduction of the selected color features for dynasty and utensil classifications in the future.

\section{CONCLUSIONS AND FUTURE WORKS}

Qiong kiln ceramics have substantial artistic value and are important Chinese ceramics. To avoid the disturbance of subjective experience, computer-aided technologies are employed to quickly automatically accurately and quantitatively process a large number of Qiong kiln ceramic images and generate the detailed statistical data. The color features of Qiong kiln ceramics are analyzed for the quantitative aesthetics. In our method, the Qiong kiln ceramic images are segmented with GrabCut algorithm. Three moments (first order, second order, and third order) of RGB and HSV components are calculated, and their DPs are analyzed according to various dynasties and various utensils, which are helpful to the selection of the discriminant color features for dynasty and utensil classifications. This paper can substantially promote the quantitative aesthetic research of Qiong kiln ceramics and is conducive to the research on the aesthetics of other ceramics.

Table 6. DPs of color moments for various dynasties.

\begin{tabular}{llll}
\hline & 1st-order & 2nd-order & 3rd-order \\
\hline $\mathrm{R}$ & $\mathbf{0 . 8 4}$ & 1.00 & 1.03 \\
$\mathrm{G}$ & 1.08 & 1.08 & 1.21 \\
$\mathrm{~B}$ & 1.15 & $\mathbf{1 . 4 5}$ & 1.34 \\
$\mathrm{H}$ & $\mathbf{0 . 8 4}$ & 1.20 & $\mathbf{0 . 8 4}$ \\
$\mathrm{S}$ & 0.95 & 1.13 & 1.07 \\
$\mathrm{~V}$ & 1.29 & 0.92 & 1.14 \\
\hline
\end{tabular}

Table 7. DPs of color moments for various utensils.

\begin{tabular}{llll}
\hline & 1st-order & 2nd-order & 3rd-order \\
\hline $\mathrm{R}$ & 1.12 & 1.17 & $\mathbf{1 . 1 9}$ \\
$\mathrm{G}$ & 1.02 & 1.05 & 1.01 \\
$\mathrm{~B}$ & 1.06 & 1.01 & 1.00 \\
$\mathrm{H}$ & $\mathbf{0 . 9 5}$ & 0.99 & 1.15 \\
$\mathrm{~S}$ & 1.13 & 1.05 & 1.04 \\
$\mathrm{~V}$ & 1.12 & 0.99 & 1.07 \\
\hline
\end{tabular}

In the future, more discriminant features, such as shape and texture, will be comprehensively analyzed to further improve the accuracy of quantitative aesthetics. In addition, we will design the dynasty and utensil classifications based on the fusion and dimensionality reduction of the discriminant features.

\section{Acknowledgement}

This research was supported by National Natural Science Foundation of China (61866028), Key Program Project of Research and Development (Jiangxi Provincial Department of Science and Technology) (20171ACE50024), Foundation of China Scholarship Council (CSC20190836007), Open Foundation of Key Laboratory of Jiangxi Province for Image Processing and Pattern Recognition (ET201680245, TX201604002), and "Triplelittle" Extracurricular Academic Projects (2019RJ072).

\section{REFERENCES}

[1] G. S. Hong, J. H. Lee, Y. W. Lee, and B. G. Kim, "New vehicle verification scheme for blind spot area based on imaging sensor system," Journal of Multimedia Information System, vol. 4, no. 1, pp. 9-18, 2017.

[2] B. G. Kim, D. J. Park, "Adaptive image normalisation based on block processing for enhancement of fingerprint image," Electronics Letters, vol. 38, no. 14, pp. 696-698, 2002.

[3] M. Kampel and M. Sablatnig, "Color classification of archaeological fragments," in Proceedings of the 15th International Conference on Pattern Recognition, pp. 771-774, Sept. 2000.

[4] L. Leng, A. B. J. Teoh, M. Li, and M. K. Khan, “A remote cancelable palmprint authentication protocol based on multi-directional two-dimensional PalmPhasorfusion," Security and Communication Networks, vol. 7, no. 11, pp. 1860-1871, 2014.

[4] L. Leng and A. B. J. Teoh, "Alignment-free row-cooccurrence cancelable palmprint Fuzzy Vault," Pattern Recognition, vol. 48, no. 7, pp. 2290-2303, 2015.

[5] L. Leng and J. S. Zhang, "PalmHash Code vs. PalmPhasor Code," Neurocomputing, vol. 108, pp. 1-12, 2013.

[6] P. Smith, D. Bespalov, and A. Shokoufandeh, "Classification of archaeological ceramic fragments using texture and color descriptors," in Proceedings of the IEEE Computer Society Conference on Computer Vision and Pattern Recognition Workshops (CVPRW), pp. 19-54, 2010.

[7] M. H. Karimi and D. Asemani, "Surface defect detection in tiling industries using digital image processing methods: Analysis and evaluation," ISA Trans, vol. 53, no. 3, pp. 834-844, 2014. 
[8] A. N. Shire, M. M. Khanapurkar, and R. S. Mundewadikar, "Plain ceramic tiles surface defect detection using image processing," in Proceedings of the 4th International Conference on Emerging Trends in Engineering \& Technology, pp. 212-220, 2011.

[9] J. A. Penaranda, C. Flores, and L. G. Corzo, "Colour and proportion of grains measurement in through-body porcelain tiles by means of image processing," Journal of the European Ceramic Society, vol. 26, no. 15, pp. 3373-3381, 2006.

[10] Z. R. Sun, "Study on the digital image processingbased dynamic detection method for ceramic disk," Journal of Transcluction Technology, vol. 18, no. 1, pp. 70-73, 2005.

[11] H. M. Elbehiery, G. Abdelmouez M. A. A. Hefnawy, and M. T. Elewa, "Surface defects detection for fired ceramic tiles using monochrome and color image processing analysis," Nov. 2004; http://econwpa.wustl.edu. (accessed on 11 July 2018)

[12] A. Ebrahimzadeh and M. Hossienzadeh, "An efficient system for automatic sorting of the ceramic tiles," in Proceedings of the 6th International Conference on Digital Content, Multimedia Technology and its Applications, pp. 372-374, Aug. 2010.

[13] L. Leng, J. S. Zhang, M. K. Khan, X. Chen, and K. Alghathbar, "Dynamic weighted discrimination power analysis: a novel approach for face and palmprint recognition in DCT domain," International Journal of the Physical Sciences, vol. 5, no. 17, pp. 2543-2554, 2010.

[14] L. Leng, J. S. Zhang, J. Xu, M. K. Khan, and K. Alghathbar, "Dynamic weighted discrimination power analysis in DCT domain for face and palmprint recognition," in Proceedings of the International Conference on Information and Communication Technology Convergence (ICTC), Seoul, pp. 467-471, Nov. 2010.

[15] G. S. Hong, B.G. Kim, D. P. Dogra, and P. P. Roy, “A survey of real-time road detection techniques using visual color sensor," Journal of Multimedia Information System, vol. 5, no. 1, pp. 9-14, 2018.

[16] B. G. Kim, J. I. Shim, and D. J. Park, "Fast image segmentation based on multi-resoluition analysis and wavelets," Pattern Recognition Letters, vol. 24, no. 16, pp. 2995-3006, 2003.

[17] C. Rother, V. Kolmogorov, and A. Blake, "GrabCut: Interactive foreground extraction using iterated graph cuts," ACM Trans. Graph, vol. 23, no. 3, pp. 309314, 2004.

[18] L. Leng, M. Li, C. Kim, and X. Bi, "Dual-source discrimination power analysis for multi-instance contactless palmprint recognition Multimedia Tools and Application," Multimedia Tools and Applications, vol. 76, no. 1, pp. 333-354, 2017.

[19] L. Leng, J. S. Zhang, G. Chen, M. K. Khan, and P. Bai, "Two dimensional PalmPhasor enhanced by multiorientation score level fusion," in Proceedings of the 8th FTRA International Conference on Secure and Trust Computing, Data Management, and Applications (STA), Crete, vol. 186, pp. 122-129, Jun. 2011.

[20] L. Leng, J. S. Zhang, G. Chen, M. K. Khan, and K. Alghathbar, "Two-directional two-dimensional random projection and its variations for face and palmprint recognition," in Proceedings of the International Conference Computational Science and Its Applications (ICCSIA), Santander, vol. 6786, pp. 458470, 2011.

[21] Byung-Gyu Kim, Dong-Jo Park, "Novel Target Segmentation and Tracking Based on Fuzzy Membership Distribution for Vision-Based Target Tracking System," Image and Vision Computing, vol. 24, no. 12, pp. 1319-1331, 2006.

Authors

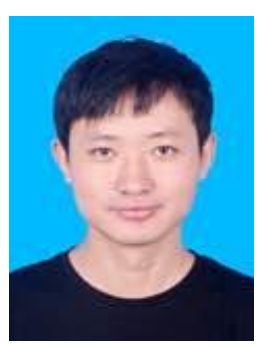

Fei Wang received his BS degree in Yunnan Agricultural University, Kunming, P. R. China in 2015. He is now pursuing his MS degree in Nanchang Hangkong University, Nanchang, P. R. China.

His research interests include pattern recognition, image processing, and deep learning.

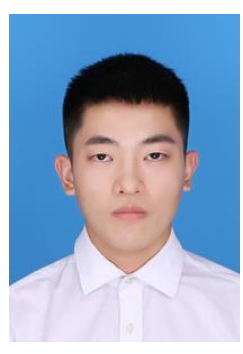

Hang Chao received his BS degree from Nanchang Hangkong University, Nanchang, P. R. China in 2019. He is currently pursuing his master degree at the University of Electronic Science and Technology of China, Chengdu, P. R. China.

His research interests include network and data security, image processing.

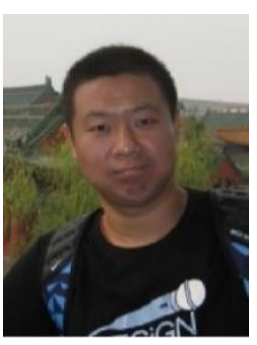

Lu Leng received his $\mathrm{Ph}$. D. degree from Southwest Jiaotong University, Chengdu, P. R. China, in 2012. He performed his post-doctoral research at Yonsei University, Seoul, Republic of Korea, and Nanjing University of Aeronautics and Astronautics, Nanjing, P. R. China from 2012 to 2015 . He was a visiting scholar at West Virginia University, USA from 2015 to 2016.

Currently, he is an associate professor at Nanchang Hangkong University, and a visiting scholar at Yonsei University, Seoul, Republic of Korea. 
Journal of Multimedia Information System VOL. 7, NO. 2, June 2020 (pp. 97-106): ISSN 2383-7632 (Online) http://doi.org/10.33851/JMIS.2020.7.2.97

He has published more than 70 international journal and conference papers. He has been granted several scholarships and funding projects for his academic research. He is the reviewer of several international journals and conferences. His research interests include computer vision, biometric template protection, and biometric recognition.

Dr. Leng is a member of Institute of Electrical and Electronics Engineers (IEEE), Association for Computing Machinery (ACM), China Society of Image and Graphics (CSIG), and China Computer Federation (CCF). 
Color Analysis for the Quantitative Aesthetics of Qiong Kiln Ceramics 